\title{
Monitoring of Crystallization Process in Solution-Processed Pentacene Thin Films by Chemical Conversion Reactions
}

\section{$\operatorname{AUTHOR}(\mathrm{S})$ :}

Shioya, Nobutaka; Fujiwara, Ryoi; Tomita, Kazutaka; Shimoaka, Takafumi; Okudaira, K. Koji; Yoshida, Hiroyuki; Koganezawa, Tomoyuki; Hasegawa, Takeshi

\section{CITATION:}

Shioya, Nobutaka ... [et al]. Monitoring of Crystallization Process in Solution-Processed Pentacene Thin Films by Chemical Conversion Reactions. The Journal of Physical Chemistry C 2021, 125(4): 2437-2445

\section{ISSUE DATE:}

2021-01-10

URL:

http://hdl.handle.net/2433/261219

\section{RIGHT:}

This document is the Accepted Manuscript version of a Published Work that appeared in final form in The Journal of Physical Chemistry C, copyright $\odot$ American Chemical Society after peer review and technical editing by the publisher To access the final edited and published work see https://doi.org/10.1021/acs.jpcc.0c10431.; The full-text file will be made open to the public on 10 January 2022 in accordance with publisher's 'Terms and Conditions for Self-Archiving' This is not the published version. Please cite only the published version.; この論文は出版社版でありません。引用の際 には出版社版をご確認ご利用ください。 


\section{Monitoring of Crystallization Process in Solution-}

\section{Processed Pentacene Thin Films by Chemical}

\section{Conversion Reactions}

Nobutaka Shioya,$^{\dagger}$ Ryoi Fujiwara,$^{\dagger}$ Kazutaka Tomita ${ }^{\dagger}$ Takafumi Shimoaka,${ }^{\dagger}$ Koji K. Okudaira,${ }^{\ddagger}$ Hiroyuki Yoshida, ${ }^{\ddagger} \S$ Tomoyuki Koganezawa," and Takeshi Hasegawa*,†

†Institute for Chemical Research, Kyoto University, Gokasho, Uji, Kyoto 611-0011, Japan

Graduate School of Engineering, Chiba University, 1-33 Yayoi-cho, Inage-ku, Chiba 263-8522, Japan

${ }^{\S}$ Molecular Chirality Research Center, Chiba University, 1-33 Yayoi-cho, Inage-ku, Chiba 2638522, Japan

IIIJapan Synchrotron Radiation Research Institute, 1-1-1 Kouto, Sayo-cho, Sayo-gun, Hyogo 6795198, Japan

\section{Corresponding Author}

* To whom correspondence should be addressed.

E-mail: htakeshi@scl.kyoto-u.ac.jp 
ABSTRACT. Solution-processable organic semiconductors having bulky substituent groups on the $\pi$-conjugated skeleton are rapidly gaining attention for their potential applications to large-area electronics. While the substituent groups contribute to the good solubility in organic solvents, they give rise to hopping sites in a thin film, affecting adversely the charge carrier transport. As an alternative material, a solvent-soluble precursor compound with thermally cleavable functional groups is promising, which can be converted by a heat treatment in a thin film to generate the desired material consisting solely of conjugated systems. This precursor approach is practically applied to various thin-film-based devices. The overall process of the thin film growth, however, remains unrevealed. In the present study, solution-processed pentacene thin films are prepared from a thermally convertible precursor, and the structural evolution during the chemical conversion reaction has been revealed by a combination of cutting-edge analytical tools of twodimensional X-ray diffraction (2D-GIXD) and p-polarized multiple-angle incidence resolution spectrometry (pMAIRS). The highlight is that pentacene is crystallized in a stepwise manner in the thermally converted films, which is substantially different from a typical growth process. In addition, influences of the oxidation reaction of pentacene on the molecular arrangement are also discussed quantitatively. This study provides a fundamental schematic of thin films grown by the precursor method. 


\section{TOC GRAPHICS}

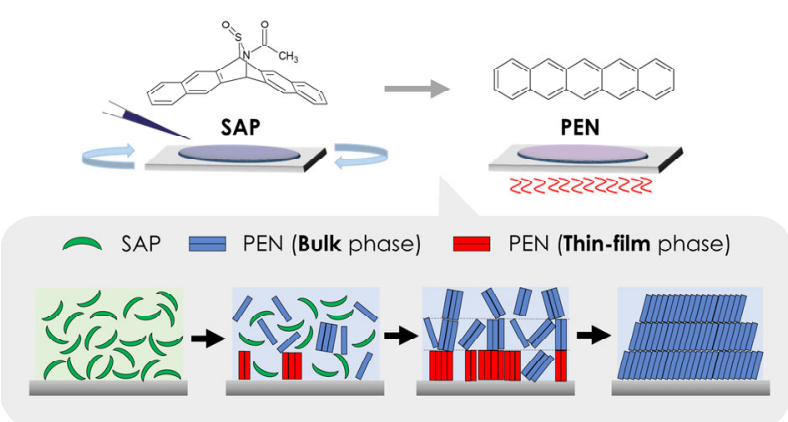

KEYWORDS. Organic semiconductors, soluble precursor, thin film growth, spin coating, X-ray diffraction, infrared spectroscopy. 


\section{INTRODUCTION}

Structural control and analysis of organic semiconductors grown on dielectric surfaces are an important subject in the field of organic electronics because of the decisive role of determination of the molecular aggregation structure for charge carrier transport in thin-film-based devices. ${ }^{1-4}$ To control the aggregation structure in terms of molecular orientation, packing, and surface morphology, analysis of the growth process depending on the deposition conditions is fundamental and essential. Solution-processable materials are particularly an important research target for industry applications, since solution-coating techniques such as spin coating, ${ }^{5}$ dip coating, ${ }^{6}$ blade coating, ${ }^{7,8}$ and inkjet printing ${ }^{9,10}$ are suitable for roll-to-roll processing and large-area electronics. ${ }^{11}$

Some semiconducting materials with high carrier mobility are insoluble in most solvents, and therefore they cannot be applied to wet processes. Instead, a physical vapor deposition (PVD) technique is frequently employed to obtain a thin film. To develop solution-processed and highperformance organic thin-film transistors (OTFTs), various soluble small-molecule organic semiconductors have recently been synthesized. ${ }^{12-18}$ The pioneering example is 6,13bis(triisopropylsilylethynyl)pentacene (TIPS-PEN), ${ }^{19}$ which has two soluble TIPS groups on the PEN core. Solution-processed OTFT devices using TIPS-PEN show a high mobility comparable to that of the conventional PEN transistors. ${ }^{20-23}$ Among many promising organic semiconducting materials, benzothieno[3,2-b][1]benzothiophene $(\mathrm{BTBT})^{16-18}$ and dinaphtho[2,3-b:2',3'f] thieno[3,2-b]thiophene (DNTT) $)^{13-15}$ derivatives show outstanding device performances in terms of carrier mobility and air stability, which exceeds those of the prototypical materials of PEN and TIPS-PEN.

Another approach to film preparation by a wet process is the precursor method. ${ }^{24-26}$ For this technique, a soluble precursor of an organic semiconductor is dissolved in common organic 
solvents, and a precursor thin film is prepared by spin coating. The precursor compound in the film is then converted to the target material by heating or photoirradiation, as reported for some insoluble semiconducting materials such as PEN, ${ }^{24-26}$ benzoporphyrins, ${ }^{24,26-28}$ phthalocyanines, ${ }^{26}$ DNTT, ${ }^{29-31}$ and rylene diimides. ${ }^{32,33}$ Although this precursor method is readily applied to various practical devices ranging from OTFTs ${ }^{29-32,34-49}$ to organic photovoltaics ${ }^{50-54}$ and perovskite solar cells,${ }^{33}$ there is still room for improvement in device performances. For example, PEN transistors fabricated by using this technique often exhibit lower carrier mobility than the conventional PEN devices. ${ }^{26}$ One of the reasons for the poor performance is the low film quality in terms of crystallinity and molecular orientation, indicating that further structural studies of the precursor method are required.

One of the most representative precursor materials is $13,6-N$-sulfinylacetamidopentacene (SAP), ${ }^{49}$ which is converted to PEN by a thermal treatment as schemed in Figure 1. Recently, we have investigated the film growth process by using infrared (IR) spectrometry. ${ }^{55}$ The results show that the structural evolution in the film occurs in two steps: 1) the thermal conversion from SAP to PEN, followed by 2) the orientation change from the random to end-on arrangement. The random orientation is responsible for the presence of unreacted SAP molecules that prevent the ordering of PEN. Under an annealing condition where the conversion reaction proceeds slowly and does not complete, as a matter of fact, the product of PEN is highly disordered in the film. ${ }^{55}$

On the other hand, for PVD, the compound is known to take a highly ordered structure immediately after depositing on the substrate. ${ }^{56-59}$ In addition, TIPS-PEN is easily crystallized even in a spin-coated film without post-annealing. ${ }^{60}$ To understand the difference of the growth process, in the present study, the structural change in the film during the thermal conversion reaction of SAP to PEN is comprehensively investigated by employing multiple analytical techniques of two- 
dimensional X-ray diffraction (2D-GIXD), atomic force microscopy (AFM), and p-polarized multiple-angle incidence resolution spectrometry ${ }^{61,62}$ (pMAIRS).

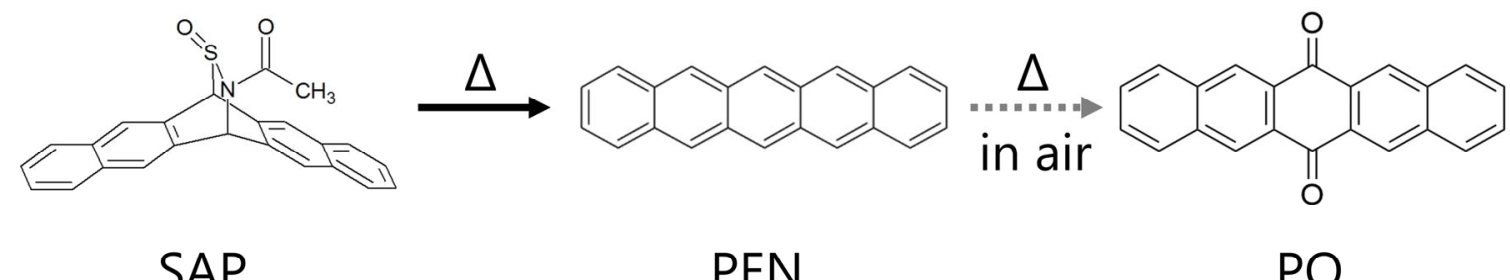

Figure 1. Structural conversion reaction of SAP to PEN followed by a natural oxidation reaction to PQ.

\section{EXPERIMENTAL SECTION}

Film Preparation. All reagents used in this study were purchased from Sigma-Aldrich (Milwaukee, WI). SAP was dissolved in chloroform at a concentration of $1.5 \mathrm{wt} \%$, and the solution was spin-coated on a silicon substrate at $1500 \mathrm{rpm}$. The film thickness of the as-spun film was estimated to be about $110 \mathrm{~nm}$ by X-ray reflectivity measurements. PEN thin films were obtained by heating the SAP spin-coated films on a pre-heated hot plate at various substrate temperatures, $T$, for $10 \mathrm{~min}$ in a nitrogen $\left(\mathrm{N}_{2}\right)$ atmosphere. The temperature of the substrate surface was measured directly using a thermocouple. The resulting films were subjected to 2D-GIXD, AFM, and pMAIRS measurements under ambient conditions.

2D-GIXD measurements. The 2D-GIXD measurements with a synchrotron radiation source were performed on a Huber (Rimsting, Germany) diffractometer at the BL46XU beamline of SPring-8 (Hyogo, Japan). A parallel X-ray beam with energy of $12.39 \mathrm{keV}(\lambda=0.1 \mathrm{~nm})$ was irradiated onto the sample surface at an incident angle of $0.12^{\circ}$ from the surface parallel. The Xray beam size was $0.4 \times 0.04 \mathrm{~mm}^{2}$ at the center of the goniometer, and the footprint on the surface 
was estimated to be $19.1 \mathrm{~mm}$. The scattered rays from the film were detected by a Dectris (Baden, Switzerland) Pilatus 300K area detector.

AFM measurements. The AFM topographic images were obtained by a Seiko Instruments Inc. (Chiba, Japan) NanoNavi IIs Probe Station equipped with a probing microscope unit, Nanocute. The measurements were performed using a silicon cantilever with a force contact of $19 \mathrm{~N} \mathrm{~m}^{-1}$ with the tapping mode.

IR pMAIRS measurements. The pMAIRS measurements were carried out on a Thermo Fischer Scientific (Madison, WI) Nicolet iS50 FT-IR spectrometer equipped with an automatic MAIRS accessory (TN 10-1500). The p-polarized ray was generated through a PIKE Technologies (Madison, WI) wire-grid linear polarizer built on germanium $(\mathrm{Ge})$. The single-beam spectra were measured by changing the angle of incidence from 9 to $44^{\circ}$ in $5^{\circ}$ steps with a wavenumber resolution of $4 \mathrm{~cm}^{-1}$, and they were used to generate the pMAIRS-IP (in-plane) and -OP (out-ofplane) spectra.

IR ATR measurements. A powder sample of 6,13-pentacenequinone (PQ) was subjected to IR attenuated total-reflection (ATR) measurements on a Thermo Fischer Scientific Magna 550 FTIR spectrometer equipped with an ATR attachment. The sample was placed on the ATR prism made of $\mathrm{Ge}$, and the spectrum was measured at a fixed angle of incidence of $45^{\circ}$. No polarizer was used for the measurement, and the wavenumber resolution was set to $4 \mathrm{~cm}^{-1}$. The band assignments were done by the density functional theory (DFT) calculation. The calculation was performed with a basis set of $6-31+G(d, p)$ using the Gaussian 09 software.

\section{RESULTS AND DISCUSSION}


Stepwise Thin Film Growth. We first discuss the molecular structure of SAP in the as-spun film prepared at room temperature $(T \approx 298 \mathrm{~K})$. As found in Figure $2 \mathrm{a}$ and $\mathrm{f}$, the film has a featureless topographic image and negligible diffraction peaks, respectively. This indicates that the precursor compound forms an amorphous structure having a flat surface in the film, which agrees with former studies. ${ }^{37,63,64}$ Note that a bulk solid of SAP is reported to be composed of polycrystalline aggregates, ${ }^{64}$ implying that the amorphous structure has a kinetic advantage in a spin-coated thin film.

(a)

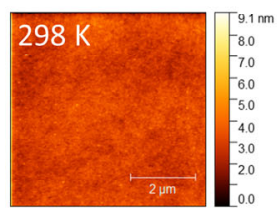

(f)

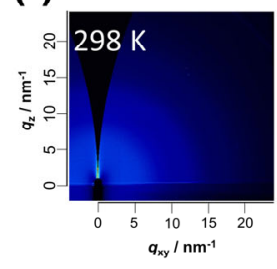

(b)

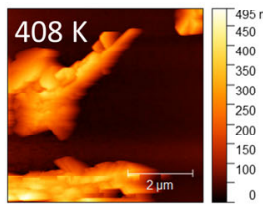

(g)

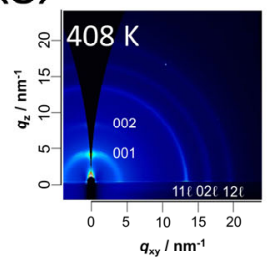

(c)

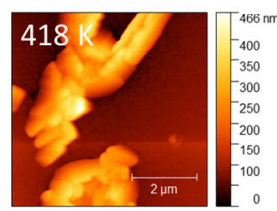

(h)

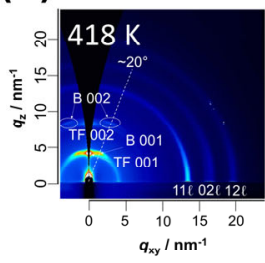

(d)

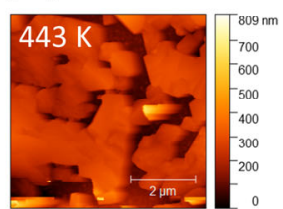

(i)

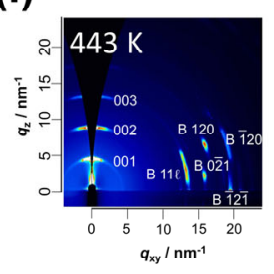

(e)

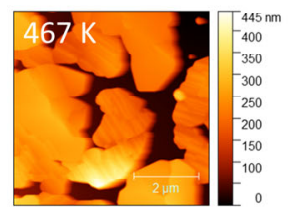

(j)

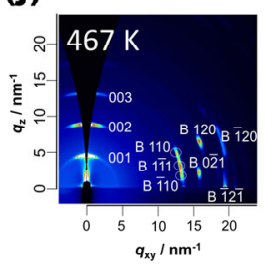

(k)

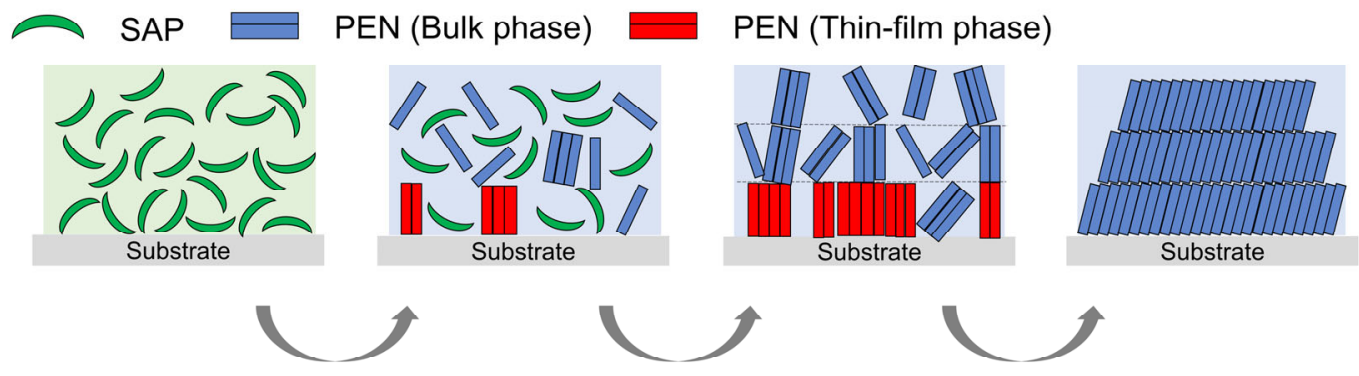

Figure 2. AFM topographic images (a-e), and 2D-GIXD patterns (f-j) of as-spun SAP and thermally converted PEN thin films on silicon prepared as a function of annealing temperature. In the diffraction patterns, "B" and "TF" denotes the bulk and thin-film phases, respectively. A schematic image of the thin film growth during the structural conversion reaction of SAP to PEN $(\mathrm{k})$. 
When the as-spun film is annealed at $T=408 \mathrm{~K}$, ring-shaped diffraction patterns of PEN appear in Figure 2g. This means that the conversion reaction of SAP to PEN occurs by at least this temperature, and the product is randomly oriented in the film. The crystalline grains are indeed observed in the AFM image (Figure 2b). The intensity and shape of the diffraction pattern change significantly as the substrate temperature is increased, as shown in Figure $2 \mathrm{~g}-\mathrm{j}$. For the film annealed at $418 \mathrm{~K}$, the $00 \ell$ peak series appear more strongly along the scattering vector in the outof-plane direction $\left(q_{z}\right)$ than the in-plane direction $\left(q_{x y}\right)$, although the other reflections $(11 \ell, 02 \ell$, and $12 \ell)$ keep the ring-shaped pattern. This mixed pattern of strong spots and ring circles is typically observed for liquid-crystalline films. ${ }^{56,65}$ Judging from the pattern, the long axis of molecule tends to stand perpendicularly on the film surface with a layer-by-layer structure, but they are not rigidly packed within the layer ${ }^{56}$ On annealing at a higher temperature, the molecular order is improved judging from the well-separated diffraction peaks in Figure $2 i$ and $j$, ${ }^{56,57,66,67}$ which meets the image of the typical oriented structure with the $c^{*}$-axis parallel to the surface normal. ${ }^{67}$ Throughout Figure 2, in this manner, the surface morphology measured by AFM has a good correlation with the diffraction patterns.

These results suggest a stepwise crystallization process of PEN in the film as depicted in Figure $2 \mathrm{k}$. Such a growth process is, as a matter of fact, rarely observed for PEN and its related compounds. For the case of vapor-deposited PEN thin films, the molecules are spontaneously ordered without annealing due to the high aggregation property. ${ }^{56-59}$ Therefore, the unique crystallization process via the random orientation found in the present work should be specific to the precursor method. We have already revealed that the self-aggregation of PEN in a thin film is disturbed by the presence of unreacted precursor molecules. ${ }^{55}$ According to the work, the precursor compound is 
mostly converted to PEN above $T=420 \mathrm{~K}$, which explains the reason why the present films prepared at 408 and $418 \mathrm{~K}$ have an unusually disordered structure.

Of additional note is that, for the film annealed at $418 \mathrm{~K}$, the diffraction peaks belong to two different polymorphs of the bulk (Campbell) $)^{68,69}$ and thin-film ${ }^{66,70,71}$ phases with the interlayer distance of 1.44 and $1.54 \mathrm{~nm}$, respectively. The $00 \ell$ reflections of the "bulk" phase appear on a tilted line at about $20^{\circ}$ from the $q_{\mathrm{z}}$ axis, while those of the "thin-film" phase are along the $q_{\mathrm{z}}$ axis (Figure $2 \mathrm{~h}$ ). This means that the (001) plane of the bulk phase is tilted by $\sim 20^{\circ}$ on the film surface, which is a characteristic of the bulk phase grown on the thin-film phase. ${ }^{56,57,72-74}$ At a higher temperature where the thin-film phase almost disappears, ${ }^{55}$ the bulk phase has no inclined structure: the $c^{*}$-axis is vertical to the substrate surface, as found in Figure $2 \mathrm{i}$ and $\mathrm{j}$. These diffraction results therefore confirm the conventional findings. ${ }^{56,57,72-74}$

Thin Film Growth in Air. Next, influences of environmental conditions of a film preparation process on the thin film growth are discussed as follows. Air stability is a long-term influential factor for PEN devices, because PEN is remarkably degraded by exposure to air. ${ }^{44,75-81}$ The compound is, in fact, known to be easily oxidized by water vapor or oxygen in air, which is accelerated by heat or light. ${ }^{82,83}$ The oxidation product gives rise to trapping sites in the film, affecting the charge carrier transport. ${ }^{84-86}$ This is a reason that the thermal conversion reaction of SAP to PEN is performed in a $\mathrm{N}_{2}$ atmosphere. Regardless, no oxidation products have been confirmed so far in thermally converted PEN films prepared under ambient condition.

Angelis and co-workers tried to identify five different oxidation products of PEN by mass spectrometry. ${ }^{82}$ Among these products, 6,13-pentacenequinone (PQ; Figure 1) is known as a main impurity in a commercial PEN reagent. ${ }^{86}$ Unfortunately, XRD is unsuitable for the detection of minute PQ. In fact, no diffraction peaks of PQ are detected even by using a synchrotron radiation 
source in thermally converted PEN thin films in air (Figure S1). Instead, IR spectroscopy is a good candidate for the purpose as demonstrated in several papers, ${ }^{86-88}$ since this technique has high sensitivity for nanometer-scale films ${ }^{89,90}$ In this work, IR pMAIRS is employed to discuss the aggregation structure of both PEN and PQ in a thin film.

Figure 3 presents the pMAIRS spectra of thermally converted PEN films under (a) air and (b) $\mathrm{N}_{2}$ conditions. Two bands at 1674 and $1281 \mathrm{~cm}^{-1}$ are observed only for the films prepared in air (Figure 3a), and they are not assigned to the vibrational modes of PEN or SAP, either. In addition, their bands are not observed at a temperature below $\sim 408 \mathrm{~K}$ (Figure S2), in which the structural conversion from SAP to PEN is partially restricted judging from the relative band intensity of PEN to SAP. These experimental facts suggest that the bands are originated from the oxidation products of PEN. By referring to previous studies, ${ }^{86-88}$ the bands at 1674 and $1281 \mathrm{~cm}^{-1}$ are definitely attributed to the $\mathrm{C}=\mathrm{O}$ anti-symmetric stretching vibration and $\mathrm{C}-\mathrm{H}$ in-plane deformation vibration (denoted as $\delta(\mathrm{C}-\mathrm{H})$ ) modes of $\mathrm{PQ}$, respectively. The band assignments are further confirmed by the DFT calculation as shown in Figure 4a. 


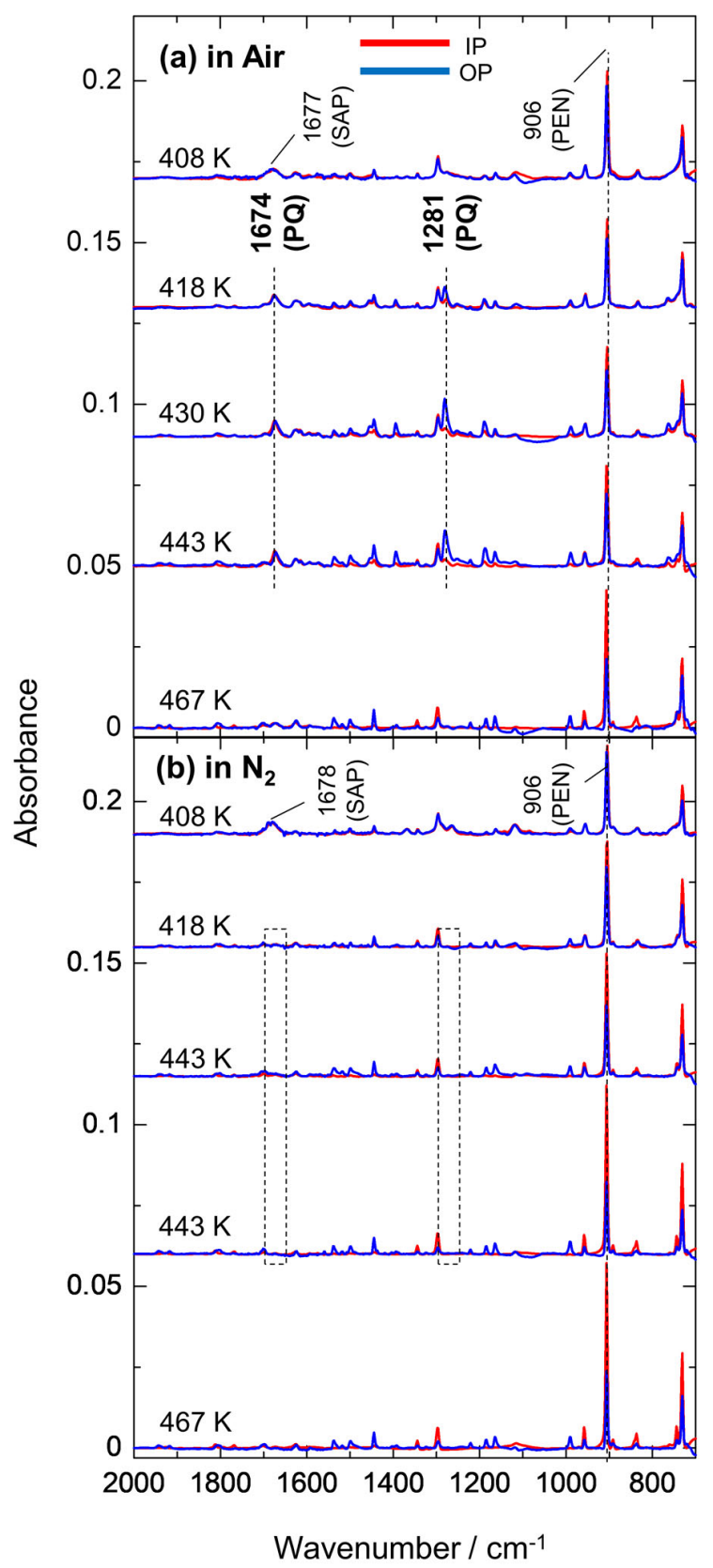

Figure 3. IR pMAIRS spectra of thermally converted PEN thin films annealed in air (a) and $\mathrm{N}_{2}$ (b) in a temperature range of $408-467 \mathrm{~K}$ for $10 \mathrm{~min}$ each. 

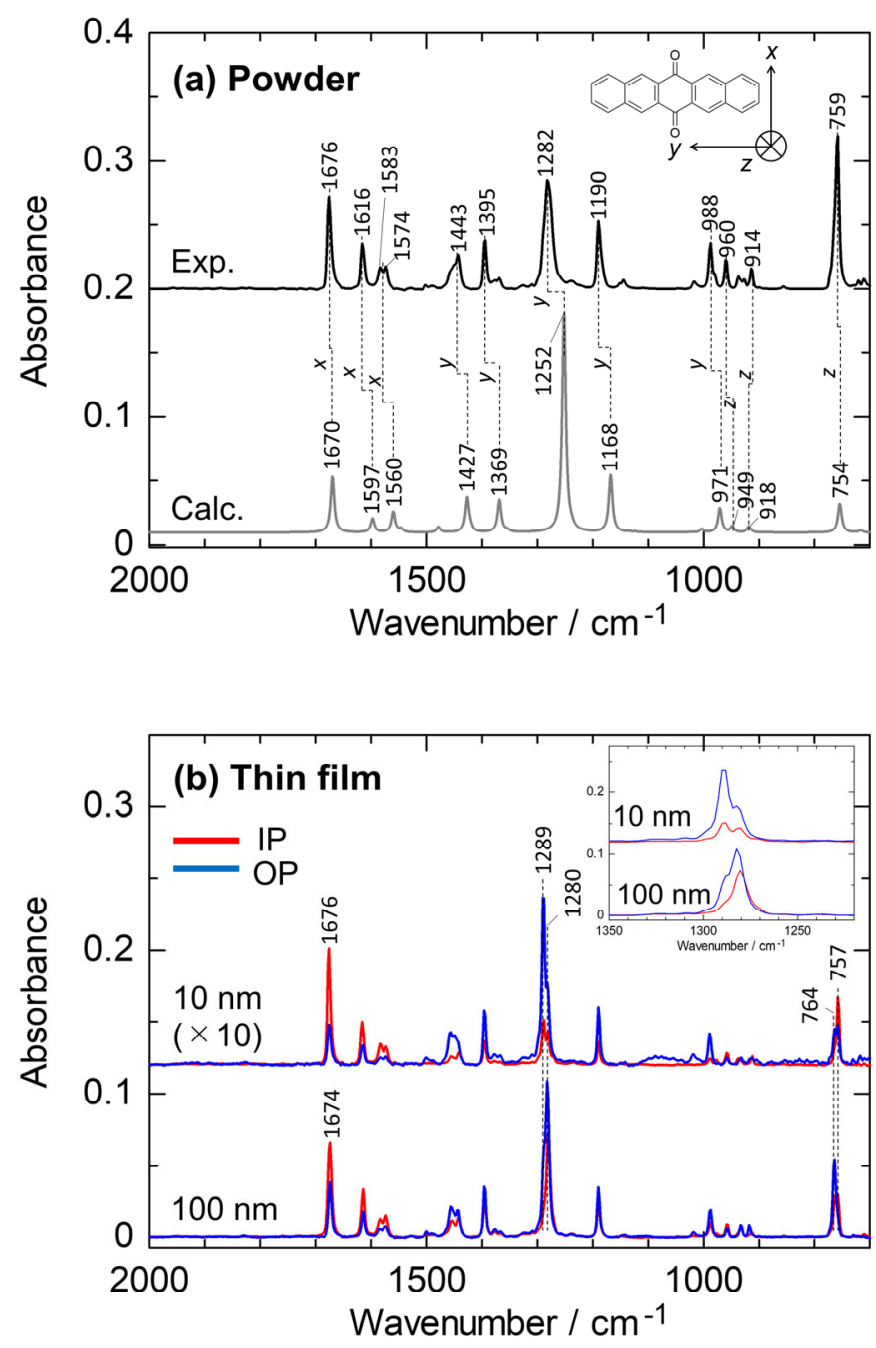

Figure 4. Experimental IR ATR spectrum of a bulk sample of PQ, and a simulated spectrum predicted by the DFT calculation with a basis set of $6-31+\mathrm{G}(\mathrm{d}, \mathrm{p})$ where wavenumbers are scaled by 0.9613 (a). IR pMAIRS spectra of vapor-deposited PQ films with a nominal thickness of 10 and $100 \mathrm{~nm}(\mathrm{~b})$. The inset in Figure 4(b) shows magnified spectra of the $\delta(\mathrm{C}-\mathrm{H})$ band.

It is noteworthy that the $\delta(\mathrm{C}-\mathrm{H})$ band of PQ in the OP spectrum is apparently stronger than that in the IP one in Figure 3a. According to the surface selection rule of MAIRS, ${ }^{61,62}$ the molecular long axis is found to stand on the film surface, since the transition dipole moment has a parallel direction to the molecular axis (Figure 4a). In general, the end-on (or edge-on) orientation of rod- 
like organic molecules is induced by intermolecular interactions on an inert surface, ${ }^{56}$ which suggests that the compound of PQ forms crystalline aggregates in the film. PQ has two crystalline polymorphs known as the bulk and thin-film phases, where the molecules in the thin-film phase do not adopt a herringbone packing structure, in contrast to the bulk phase. ${ }^{87}$ Salzmann and coworkers report that the thin-film and bulk phases give the $\delta(\mathrm{C}-\mathrm{H})$ band at different positions of $\sim 1290$ and $\sim 1280 \mathrm{~cm}^{-1}$, respectively. ${ }^{87,88}$ To check the band assignments, vapor-deposited films of PQ with a nominal thickness of 10 or $100 \mathrm{~nm}$ were subjected to pMAIRS measurements. The spectra show that the thinner film has the high-wavenumber band due to the thin-film phase as a major component, whereas the low-wavenumber band corresponding to the bulk phase is the dominant component in the thicker film (Figure $4 \mathrm{~b}$ ). This observation agrees well with the analytical results by $\mathrm{XRD}^{91,92}$ and supports the assignments proposed by Salzmann et al. On the band assignments, the "bulk" phase of PQ is concluded to be included as a minute fraction in the present films.

Of interest is that the contribution of PQ is nearly suppressed at the elevated temperature of 467 $\mathrm{K}$, as shown in Figure 3a. The pMAIRS spectra of this film show a large intensity ratio of IP to OP bands, as typically found for the most intense band of PEN at $906 \mathrm{~cm}^{-1}$, which indicates that the PEN molecule is highly oriented. The textured structure is also evidenced by the 2D-GIXD pattern (Figure S1). These results lead us to an expectation that the oxidation reaction is suppressed by highly ordered aggregates of PEN, and this interpretation is actually accepted by some papers. ${ }^{83,93}$ Indeed, as found in the pMAIRS spectra of Figure 5a and S3, most of the PEN molecules in the film remain unchanged even after re-annealing at $396 \mathrm{~K}$ for 24 hours in air. 

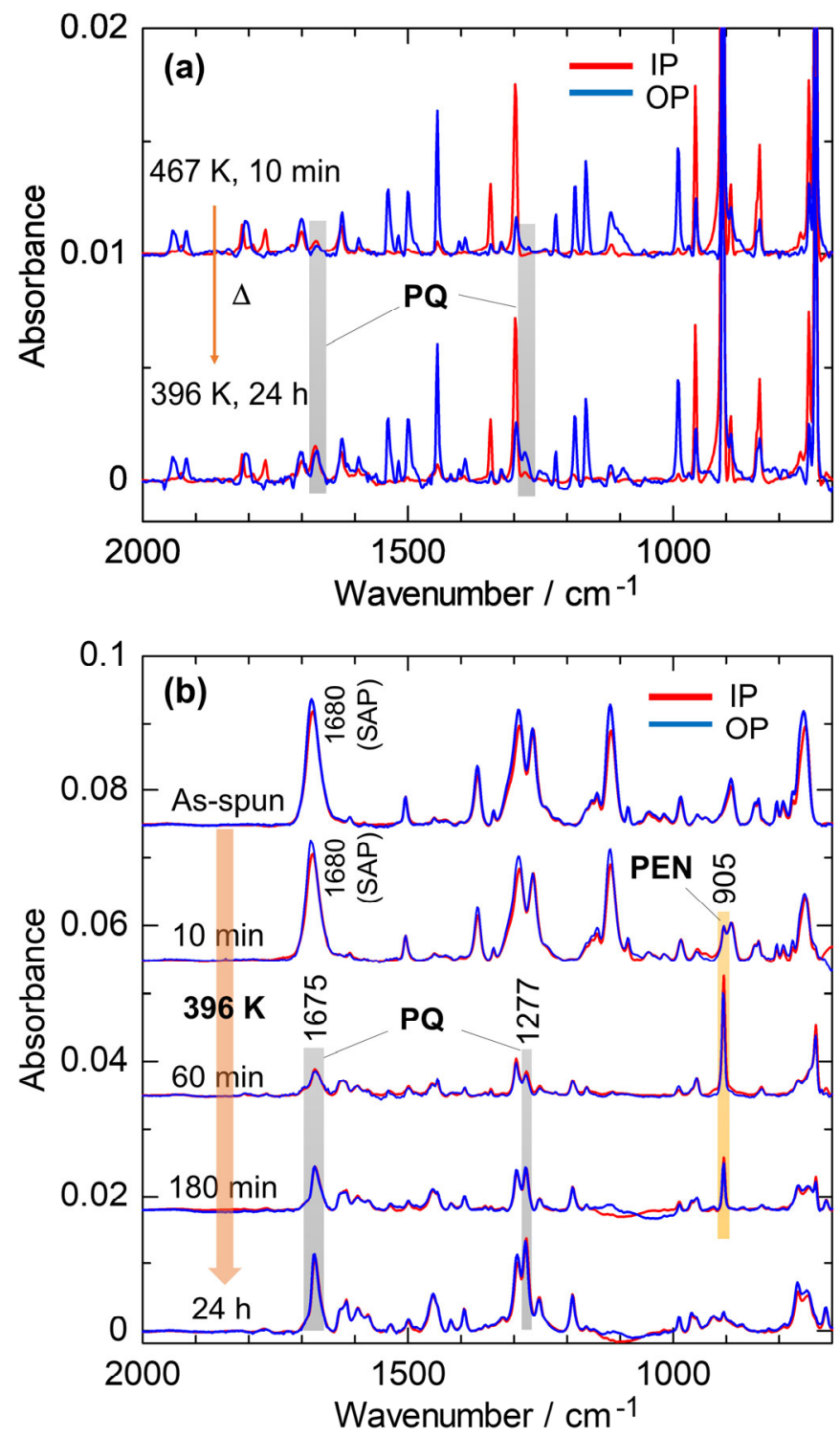

Figure 5. IR pMAIRS spectra of a thermally converted PEN thin film at the annealing temperature of $467 \mathrm{~K}$ (top spectra of (a)). After storing the film in ambient air, it was re-annealed for 24 hours at $396 \mathrm{~K}$. The spectra of the re-annealed film are shown at the bottom of (a). IR pMAIRS spectra of as-spun and thermal-annealed thin films prepared as a function of annealing time at $396 \mathrm{~K}(\mathrm{~b})$.

On the other hand, when the "as-spun" film of SAP is annealed under the same condition, the oxidation reaction proceeds drastically. The reaction depending on the annealing time is pursued 
by using pMAIRS as presented in Figure 5b. The time-dependent spectral change shows that SAP begins to be converted to PEN at about $10 \mathrm{~min}$, and the reaction is completed up to $60 \mathrm{~min}$. At this time, the oxidation product of PQ coexists with PEN. The relative concentration of PQ to PEN increases with time of annealing. It should be noted that the band intensity of PEN decreases with keeping the intensity ratio of IP and OP spectra, as apparently found for the band at $905 \mathrm{~cm}^{-1}$. This indicates that PEN keeps the random orientation in the films during the oxidation reaction. In other words, at $T=396 \mathrm{~K}$, where the thermal conversion reaction of SAP proceeds slowly (Figure $5 \mathrm{~b}$ ), the self-aggregation of PEN is always suppressed by the presence of SAP and PQ. This observation is in good agreement with the discussion above; thus, loosely packed aggregates are readily oxidized.

To investigate the molecular orientation of PEN in the mixed films, three vibrational modes with mutually orthogonal transition moments are analyzed from the pMAIRS spectra in Figure 3. The calculation procedure is referred to our previous works. ${ }^{55,61}$ Figure 6 plots the obtained orientation angles against the annealing temperature, where $\phi_{x}, \phi_{y}$, and $\phi_{z}$ correspond to the inclination angles of the $x, y$, and $z$ molecular axes (see Figure 6a) to the surface normal. The dashed line in the figure shows the magic angle of $54.7^{\circ}$, which is a guide for judging whether the analyte is oriented or not. As discussed elsewhere, ${ }^{55}$ the plot indicates a change from random to end-on molecular orientation with increasing the annealing temperature. 
(a)

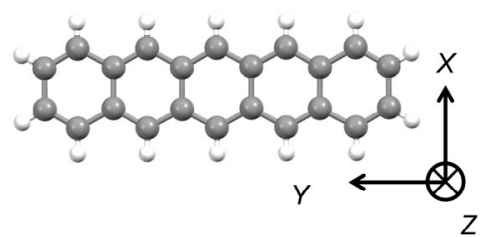

(c)

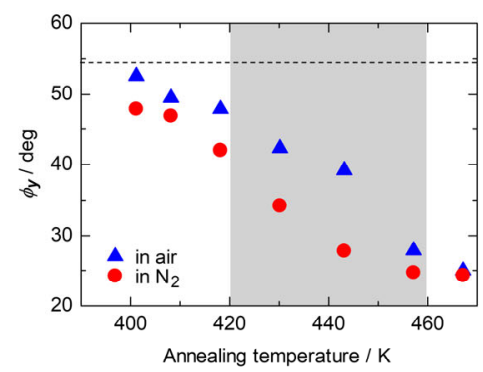

(b)

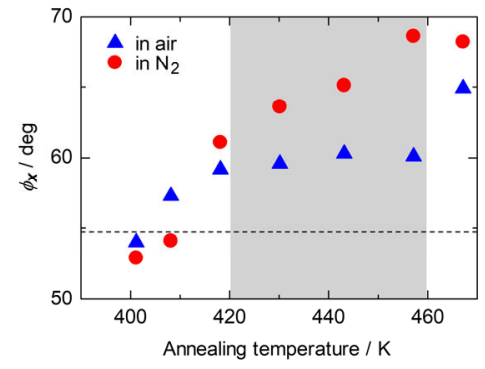

(d)

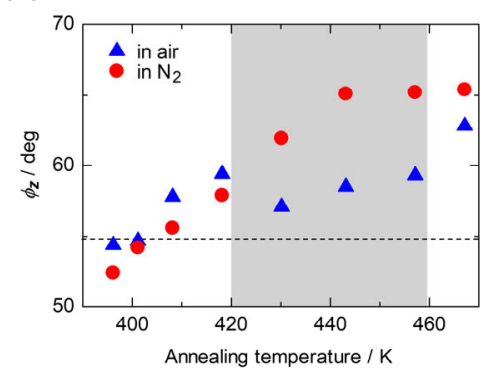

Figure 6. Average orientation angles of PEN calculated from the pMAIRS spectra in Figure 3, where $\phi_{x}$ (b), $\phi_{y}$ (c), and $\phi_{z}$ (d) are defined as the inclination angles of the $x, y$, and $z$ molecular axes (see (a)) with respect to the substrate normal.

For the films prepared in air, the angles are obviously closer to the magic angle than those obtained under a $\mathrm{N}_{2}$ environment in a specific temperature range of $420 \mathrm{~K} \lesssim T \lesssim 460 \mathrm{~K}$. The pronounced mosaicity is corroborated by the 2D-GIXD patterns (Figure S1). The difference of the molecular orientation should be correlated with the oxidation reaction of PEN, since the oxide is produced mainly in this temperature region as discussed above. Thus, the presence of PQ reduces the degree of molecular orientation of PEN as well as the crystallinity and the grain size (see Figures S1 and S4). This result is reminiscent of another case that the unconverted SAP molecules disturb the ordering of PEN in the film. Therefore, PQ would play the same role as SAP in the PEN films. Another interpretation is that the oxidation reaction has a kinetic advantage compared to the crystallization of PEN under this annealing condition. In this manner, these results provide structural evidence for a significant reduction of carrier mobility due to the oxide formation. 


\section{CONCLUSION}

The complex growth process of solution-processed PEN thin films, including the structural conversion reaction from SAP to PEN, has comprehensively been understood. The crystallization process of PEN is monitored by means of 2D-GIXD with a synchrotron radiation source. The diffraction patterns demonstrate stepwise crystal growth with respect to the crystal axes: the $c^{*}$ axis is first arranged parallel to the surface normal, and then the other two axes are aligned to form a textured thin film. The thin film growth is affected by the oxidation reaction of PEN that occurs in air. The minute oxidation product is identified by using the pMAIRS technique. The oxidation reaction is found at a relatively low temperature, where PEN is randomly oriented because of the presence of unconverted SAP molecules. The annealing process at a higher temperature, on the other hand, immediately induces a well-ordered structure of PEN, which is robust to the following oxidation reaction. Furthermore, the spectra quantitatively reveal that the presence of PQ disturbs molecular orientation of PEN.

\section{ASSOCIATED CONTENT}

\section{Supporting Information.}

The Supporting Information is available free of charge.

Additional 2D-GIXD, pMAIRS, and AFM data (PDF)

\section{AUTHOR INFORMATION}

\section{Corresponding Author}

*E-mail: htakeshi@scl.kyoto-u.ac.jp 


\section{ORCID}

Nobutaka Shioya: 0000-0002-2915-894X

Takafumi Shimoaka: 0000-0001-9131-9831

Hiroyuki Yoshida: 0000-0002-8889-324X

Takeshi Hasegawa: 0000-0001-5574-9869

\section{Notes}

The authors declare no competing financial interests.

\section{ACKNOWLEDGMENT}

This work was financially supported by a Grant-in-Aid for Scientific Research (A) (No. 15H02185 (TH)), Grant-in-Aid for Young Scientists (B) (No. 17K14502 (TS)) and Grant-in-Aid for EarlyCareer Scientists (No. 19K15602 (NS)) from the Japan Society for the Promotion of Science (JSPS), for which we are thankful. The 2D-GIXD measurements were performed at the BL46XU beamline of SPring-8 with the approval of the Japan Synchrotron Radiation Research Institute (JASRI) (Proposal No. 2018B1595). The X-ray reflectivity measurements were performed at Kobe University by courtesy of Prof. Kazuo Eda. 


\section{REFERENCES}

1. Yamamura, A.; Fujii, H.; Ogasawara, H.; Nordlund, D.; Takahashi, O.; Kishi, Y.; Ishii, H.; Kobayashi, N.; Niitsu, N.; Blülle, B.; et al. Sub-Molecular Structural Relaxation at a Physisorbed Interface with Monolayer Organic Single-Crystal Semiconductors. Commun. Phys. 2020, 3 (1), 20.

2. Zhang, Y.; Qiao, J.; Gao, S.; Hu, F.; He, D.; Wu, B.; Yang, Z.; Xu, B.; Li, Y.; Shi, Y.; et al. Probing Carrier Transport and Structure-Property Relationship of Highly Ordered Organic Semiconductors at the Two-Dimensional Limit. Phys. Rev. Lett. 2016, 116 (1), 016602.

3. Mas-Torrent, M.; Rovira, C. Role of Molecular Order and Solid-State Structure in Organic Field-Effect Transistors. Chem. Rev. 2011, 111 (8), 4833-4856.

4. Wakabayashi, Y.; Takeya, J.; Kimura, T. Sub-Å Resolution Electron Density Analysis of the Surface of Organic Rubrene Crystals. Phys. Rev. Lett. 2010, 104 (6), 066103.

5. Yuan, Y.; Giri, G.; Ayzner, A. L.; Zoombelt, A. P.; Mannsfeld, S. C. B.; Chen, J.; Nordlund, D.; Toney, M. F.; Huang, J.; Bao, Z. Ultra-High Mobility Transparent Organic Thin Film Transistors Grown by an off-Centre Spin-Coating Method. Nat. Commun. 2014, 5 (1), 3005.

6. Wu, H.; Iino, H.; Hanna, J. Scalable Ultrahigh-Speed Fabrication of Uniform Polycrystalline Thin Films for Organic Transistors. ACS Appl. Mater. Interfaces 2020, 12 (26).

7. Niazi, M. R.; Li, R.; Li, E. Q.; Kirmani, A. R.; Abdelsamie, M.; Wang, Q.; Pan, W.; Payne, M. M.; Anthony, J. E.; Smilgies, D.-M.; et al. Solution-Printed Organic Semiconductor Blends Exhibiting Transport Properties on Par with Single Crystals. Nat. Commun. 2015, 6 (1), 8598. 
8. Diao, Y.; Tee, B. C.-K.; Giri, G.; Xu, J.; Kim, D. H.; Becerril, H. A.; Stoltenberg, R. M.; Lee,

T. H.; Xue, G.; Mannsfeld, S. C. B.; et al. Solution Coating of Large-Area Organic Semiconductor Thin Films with Aligned Single-Crystalline Domains. Nat. Mater. 2013, 12 (7), 665-671.

9. Minemawari, H.; Yamada, T.; Matsui, H.; Tsutsumi, J.; Haas, S.; Chiba, R.; Kumai, R.; Hasegawa, T. Inkjet Printing of Single-Crystal Films. Nature 2011, 475 (7356), 364-367.

10. Singh, M.; Haverinen, H. M.; Dhagat, P.; Jabbour, G. E. Inkjet Printing—Process and Its Applications. Adv. Mater. 2010, 22 (6), 673-685.

11. Arias, A. C.; MacKenzie, J. D.; McCulloch, I.; Rivnay, J.; Salleo, A. Materials and Applications for Large Area Electronics: Solution-Based Approaches. Chem. Rev. 2010, 110 (1), 3-24.

12. Okamoto, T.; Mitani, M.; Yu, C. P.; Mitsui, C.; Yamagishi, M.; Ishii, H.; Watanabe, G.; Kumagai, S.; Hashizume, D.; Tanaka, S.; et al. Alkyl-Substituted Selenium-Bridged VShaped Organic Semiconductors Exhibiting High Hole Mobility and Unusual Aggregation Behavior. J. Am. Chem. Soc. 2020, 142 (35), 14974-14984.

13. Yamaguchi, Y.; Kojiguchi, Y.; Kawata, S.; Mori, T.; Okamoto, K.; Tsutsui, M.; Koganezawa, T.; Katagiri, H.; Yasuda, T. Solution-Processable Organic Semiconductors Featuring SShaped Dinaphthothienothiophene (S-DNTT): Effects of Alkyl Chain Length on SelfOrganization and Carrier Transport Properties. Chem. Mater. 2020, 32 (12), 5350-5360. 
14. Bilgaiyan, A.; Cho, S.-I.; Abiko, M.; Watanabe, K.; Mizukami, M. Solution Processed Organic Transistors on Polymeric Gate Dielectric with Mobility Exceeding $15 \mathrm{~cm}^{2} \mathrm{~V}^{-1} \mathrm{~S}^{-1}$. Phys. Status Solidi RPL 2020, 14 (8), 2000156.

15. Kawabata, K.; Usui, S.; Takimiya, K. Synthesis of Soluble Dinaphtho[2,3-b:2',3'f]thieno[3,2-b]thiophene (DNTT) Derivatives: One-Step Functionalization of 2-BromoDNTT. J. Org. Chem. 2020, 85 (1), 195-206.

16. Iino, H.; Usui, T.; Hanna, J. Liquid Crystals for Organic Thin-Film Transistors. Nat. Commun. 2015, $6(1), 6828$.

17. Amin, A. Y.; Khassanov, A.; Reuter, K.; Meyer-Friedrichsen, T.; Halik, M. Low-Voltage Organic Field Effect Transistors with a 2-Tridecyl[1]benzothieno[3,2-b][1]benzothiophene Semiconductor Layer. J. Am. Chem. Soc. 2012, 134 (40), 16548-16550.

18. Ebata, H.; Izawa, T.; Miyazaki, E.; Takimiya, K.; Ikeda, M.; Kuwabara, H.; Yui, T. Highly Soluble [1]Benzothieno[3,2-b]benzothiophene (BTBT) Derivatives for High-Performance, Solution-Processed Organic Field-Effect Transistors. J. Am. Chem. Soc. 2007, 129 (51), $15732-15733$.

19. Anthony, J. E.; Brooks, J. S.; Eaton, D. L.; Parkin, S. R. Functionalized Pentacene: Improved Electronic Properties from Control of Solid-State Order. J. Am. Chem. Soc. 2001, 123 (38), $9482-9483$.

20. Kim, D. H.; Lee, D. Y.; Lee, H. S.; Lee, W. H.; Kim, Y. H.; Han, J. I.; Cho, K. High-Mobility Organic Transistors Based on Single-Crystalline Microribbons of Triisopropylsilylethynyl Pentacene via Solution-Phase Self-Assembly. Adv. Mater. 2007, 19 (5), 678-682. 
21. Park, S. K.; Jackson, T. N.; Anthony, J. E.; Mourey, D. A. High Mobility Solution Processed 6,13-Bis(Triisopropyl-Silylethynyl) Pentacene Organic Thin Film Transistors. Appl. Phys. Lett. 2007, 91 (6), 063514.

22. Payne, M. M.; Parkin, S. R.; Anthony, J. E.; Kuo, C.-C.; Jackson, T. N. Organic Field-Effect Transistors from Solution-Deposited Functionalized Acenes with Mobilities as High as 1 $\mathrm{cm}^{2} / \mathrm{V} \cdot \mathrm{s} . J$. Am. Chem. Soc. 2005, 127 (14).

23. Sheraw, C. D.; Jackson, T. N.; Eaton, D. L.; Anthony, J. E. Functionalized Pentacene Active Layer Organic Thin-Film Transistors. Adv. Mater. 2003, 15 (23), 2009-2011.

24. Yamada, H.; Kuzuhara, D.; Suzuki, M.; Hayashi, H.; Aratani, N. Synthesis and Morphological Control of Organic Semiconducting Materials Using the Precursor Approach. Bull. Chem. Soc. Jpn. 2020, 93 (10), 1234-1267.

25. Suzuki, M.; Aotake, T.; Yamaguchi, Y.; Noguchi, N.; Nakano, H.; Nakayama, K.; Yamada, H. Synthesis and Photoreactivity of $\alpha$-Diketone-Type Precursors of Acenes and Their Use in Organic-Device Fabrication. J. Photochem. Photobiol. C 2014, 18, 50-70.

26. Yamada, H.; Okujima, T.; Ono, N. Organic Semiconductors Based on Small Molecules with Thermally or Photochemically Removable Groups. Chem. Commun. 2008, 26, 2957.

27. Okujima, T.; Hashimoto, Y.; Furuta, T.; Yamanaka, R.; Jin, G.; Otsubo, S.; Aramaki, S.; Mori, S.; Yamada, H.; Uno, H.; et al. Synthesis of the Soluble Precursors of Tetrabenzoporphyrin: Control of the Solubility and the Conversion Temperature. Bull. Chem. Soc. Jpn. 2019, 92 (8), $1370-1378$. 
28. Carvalho, C. M. B.; Brocksom, T. J.; Oliveira, K. T. de. Tetrabenzoporphyrins: Synthetic Developments and Applications. Chem. Soc. Rev. 2013, 42 (8), 3302-3317.

29. Hamaguchi, A.; Negishi, T.; Kimura, Y.; Ikeda, Y.; Takimiya, K.; Bisri, S.; Iwasa, Y.; Shiro, T. Single-Crystal-Like Organic Thin-Film Transistors Fabricated from Dinaphtho[2,3-b:2',3'f]Thieno[3,2-b]Thiophene (DNTT) Precursor-Polystyrene Blends. Adv. Mater. 2015, 27 (42), $6606-6611$.

30. Kimura, Y.; Nagase, T.; Kobayashi, T.; Hamaguchi, A.; Ikeda, Y.; Shiro, T.; Takimiya, K.; Naito, H. Soluble Organic Semiconductor Precursor with Specific Phase Separation for HighPerformance Printed Organic Transistors. Adv. Mater. 2015, 27 (4), 727-732.

31. Soeda, J.; Okamoto, T.; Hamaguchi, A.; Ikeda, Y.; Sato, H.; Yamano, A.; Takeya, J. TwoDimensional Crystal Growth of Thermally Converted Organic Semiconductors at the Surface of Ionic Liquid and High-Mobility Organic Field-Effect Transistors. Org. Electron. 2013, 14 (4), 1211-1217.

32. Hayakawa, S.; Matsuo, K.; Yamada, H.; Fukui, N.; Shinokubo, H. Dinaphthothiepine Bisimide and Its Sulfoxide: Soluble Precursors for Perylene Bisimide. J. Am. Chem. Soc. 2020, $142(27), 11663-11668$

33. Nakamura, T.; Shioya, N.; Shimoaka, T.; Nishikubo, R.; Hasegawa, T.; Saeki, A.; Murata, Y.; Murdey, R.; Wakamiya, A. Molecular Orientation Change in Naphthalene Diimide Thin Films Induced by Removal of Thermally Cleavable Substituents. Chem. Mater. 2019, 31 (5), 1729-1737. 
34. Nakayama, K.; Ohashi, C.; Oikawa, Y.; Motoyama, T.; Yamada, H. Characterization and Field-Effect Transistor Performance of Printed Pentacene Films Prepared by Photoconversion of a Soluble Precursor. J. Mater. Chem. C 2013, 1 (39), 6244-6251.

35. Yamada, H.; Ohashi, C.; Aotake, T.; Katsuta, S.; Honsho, Y.; Kawano, H.; Okujima, T.; Uno, H.; Ono, N.; Seki, S.; et al. FET Performance and Substitution Effect on 2,6Dithienylanthracene Devices Prepared by Photoirradiation of Their Diketone Precursors. Chem. Commun. 2012, 48 (90), 11136-11138.

36. Shea, P. B.; Yamada, H.; Ono, N.; Kanicki, J. Solution-Processed Zinc Tetrabenzoporphyrin Thin-Films and Transistors. Thin Solid Films 2012, 520 (11), 4031-4035.

37. Luzio, A.; Musumeci, C.; Newman, C. R.; Facchetti, A.; Marks, T. J.; Pignataro, B. Enhanced Thin-Film Transistor Performance by Combining 13,6-N-Sulfinylacetamidopentacene with Printed PEDOT:PSS Electrodes. Chem. Mater. 2011, 23 (4), 1061-1069.

38. Hirao, A.; Akiyama, T.; Okujima, T.; Yamada, H.; Uno, H.; Sakai, Y.; Aramaki, S.; Ono, N. Soluble Precursors of 2,3-Naphthalocyanine and Phthalocyanine for Use in Thin Film Transistors. Chem. Commun. 2008, 39, 4714-4716.

39. Shea, P. B.; Chen, C.; Kanicki, J.; Pattison, L. R.; Petroff, P.; Yamada, H.; Ono, N. Polycrystalline Tetrabenzoporphyrin Organic Field-Effect Transistors with Nanostructured Channels. Appl. Phys. Lett. 2007, 90 (23), 233107.

40. Shea, P. B.; Pattison, L. R.; Kawano, M.; Chen, C.; Chen, J.; Petroff, P.; Martin, D. C.; Yamada, H.; Ono, N.; Kanicki, J. Solution-Processed Polycrystalline Copper Tetrabenzoporphyrin Thin-Film Transistors. Synth. Met. 2007, 157 (4-5), 190-197. 
41. Shea, P. B.; Kanicki, J.; Pattison, L. R.; Petroff, P.; Kawano, M.; Yamada, H.; Ono, N. Solution-Processed Nickel Tetrabenzoporphyrin Thin-Film Transistors. J. Appl. Phys. 2006, $100(3), 034502$.

42. Tulevski, G. S.; Miao, Q.; Afzali, A.; Graham, T. O.; Kagan, C. R.; Nuckolls, C. Chemical Complementarity in the Contacts for Nanoscale Organic Field-Effect Transistors. J. Am. Chem. Soc. 2006, $128(6), 1788-1789$.

43. Chen, K.-Y.; Hsieh, H.-H.; Wu, C.-C.; Hwang, J.-J.; Chow, T. J. A New Type of Soluble Pentacene Precursor for Organic Thin-Film Transistors. Chem. Commun. 2006, 10, 10651067.

44. Kagan, C. R.; Afzali, A.; Graham, T. O. Operational and Environmental Stability of Pentacene Thin-Film Transistors. Appl. Phys. Lett. 2005, 86 (19), 193505.

45. Aramaki, S.; Sakai, Y.; Ono, N. Solution-Processible Organic Semiconductor for Transistor Applications: Tetrabenzoporphyrin. Appl. Phys. Lett. 2004, 84 (12), 2085-2087.

46. Murphy, A. R.; Fréchet, J. M. J.; Chang, P.; Lee, J.; Subramanian, V. Organic Thin Film Transistors from a Soluble Oligothiophene Derivative Containing Thermally Removable Solubilizing Groups. J. Am. Chem. Soc. 2004, 126 (6), 1596-1597.

47. Weidkamp, K. P.; Afzali, A.; Tromp, R. M.; Hamers, R. J. A Photopatternable Pentacene Precursor for Use in Organic Thin-Film Transistors. J. Am. Chem. Soc. 2004, 126 (40), $12740-12741$ 
48. Afzali, A.; Dimitrakopoulos, C. D.; Graham, T. O. Photosensitive Pentacene Precursor: Synthesis, Photothermal Patterning, and Application in Thin-Film Transistors. Adv. Mater. 2003, 15 (24), 2066-2069.

49. Afzali, A.; Dimitrakopoulos, C. D.; Breen, T. L. High-Performance, Solution-Processed Organic Thin Film Transistors from a Novel Pentacene Precursor. J. Am. Chem. Soc. 2002, $124(30), 8812-8813$.

50. Takahashi, K.; Kumagai, D.; Yamada, N.; Kuzuhara, D.; Yamaguchi, Y.; Aratani, N.; Koganezawa, T.; Koshika, S.; Yoshimoto, N.; Masuo, S.; et al. Side-Chain Engineering in a Thermal Precursor Approach for Efficient Photocurrent Generation. J. Mater. Chem. A 2017, $5(27), 14003-14011$.

51. Saeki, H.; Kurimoto, O.; Nakaoka, H.; Misaki, M.; Kuzuhara, D.; Yamada, H.; Ishida, K.; Ueda, Y. Effect of Crystallinity in Small Molecular Weight Organic Heterojunction Solar Cells. J. Mater. Chem. C 2014, 2 (27), 5357-5364.

52. Motoyama, T.; Kiyota, T.; Yamada, H.; Nakayama, K. Hetero-Layered Organic Photovoltaic Devices Fabricated Using Soluble Pentacene Photoprecursors. Sol. Energy Mater. Sol. Cells 2013, $114,156-160$.

53. Chen, T. L.; Chen, J. J.-A.; Catane, L.; Ma, B. Fully Solution Processed P-i-n Organic Solar Cells with an Industrial Pigment - Quinacridone. Org. Electron. 2011, 12 (7), 1126-1131.

54. Matsuo, Y.; Sato, Y.; Niinomi, T.; Soga, I.; Tanaka, H.; Nakamura, E. Columnar Structure in Bulk Heterojunction in Solution-Processable Three-Layered p-i-n Organic Photovoltaic 
Devices Using Tetrabenzoporphyrin Precursor and Silylmethyl[60]Fullerene. J. Am. Chem. Soc. 2009, 131 (44), 16048-16050.

55. Shioya, N.; Fujiwara, R.; Tomita, K.; Shimoaka, T.; Hasegawa, T. Simultaneous Analysis of Molecular Orientation and Quantity Change of Constituents in a Thin Film Using pMAIRS. J. Phys. Chem. A 2020, 124 (13), 2714-2720.

56. Shioya, N.; Murdey, R.; Nakao, K.; Yoshida, H.; Koganezawa, T.; Eda, K.; Shimoaka, T.; Hasegawa, T. Alternative Face-on Thin Film Structure of Pentacene. Sci. Rep. 2019, 9 (1), 579.

57. Watanabe, T.; Hosokai, T.; Koganezawa, T.; Yoshimoto, N. In Situ Real-Time X-Ray Diffraction During Thin Film Growth of Pentacene. Mol. Cryst. Liq. Cryst. 2012, 566 (1), 18 21.

58. Kowarik, S.; Gerlach, A.; Leitenberger, W.; Hu, J.; Witte, G.; Wöll, C.; Pietsch, U.; Schreiber, F. Energy-Dispersive X-Ray Reflectivity and GID for Real-Time Growth Studies of Pentacene Thin Films. Thin Solid Films 2007, 515 (14), 5606-5610.

59. Ruiz, R.; Choudhary, D.; Nickel, B.; Toccoli, T.; Chang, K.-C.; Mayer, A. C.; Clancy, P.; Blakely, J. M.; Headrick, R. L.; Iannotta, S.; et al. Pentacene Thin Film Growth. Chem. Mater. 2004, $16(23), 4497-4508$.

60. Chou, K. W.; Khan, H. U.; Niazi, M. R.; Yan, B.; Li, R.; Payne, M. M.; Anthony, J. E.; Smilgies, D.-M.; Amassian, A. Late Stage Crystallization and Healing during Spin-Coating Enhance Carrier Transport in Small-Molecule Organic Semiconductors. J. Mater. Chem. C 2014, 2 (28), 5681-5689. 
61. Hasegawa, T.; Shioya, N. MAIRS: Innovation of Molecular Orientation Analysis in a Thin Film. Bull. Chem. Soc. Jpn. 2020, 93 (9), 1127-1138.

62. Hasegawa, T. Advanced Multiple-Angle Incidence Resolution Spectrometry for Thin-Layer Analysis on a Low-Refractive-Index Substrate. Anal. Chem. 2007, 79 (12), 4385-4389.

63. Murai, Y.; Misaki, M.; Ishida, K.; Ueda, Y. Pillarlike Crystals of Pentacene Prepared from Soluble Precursor. Appl. Phys. Express 2011, 4 (12), 121603.

64. Akinaga, T.; Yasutake, S.; Sasaki, S.; Sakata, O.; Otsuka, H.; Takahara, A. Analysis of Molecular Aggregation States in Pentacene Thin Films Prepared from Soluble Precursor. Chem. Lett. 2006, 35 (10), 1162-1163.

65. Bishop, C.; Thelen, J. L.; Gann, E.; Toney, M. F.; Yu, L.; DeLongchamp, D. M.; Ediger, M. D. Vapor Deposition of a Nonmesogen Prepares Highly Structured Organic Glasses. Proc. Natl. Acad. Sci. U.S.A. 2019, 116 (43), 21421-21426.

66. Yoshida, H.; Inaba, K.; Sato, N. X-Ray Diffraction Reciprocal Space Mapping Study of the Thin Film Phase of Pentacene. Appl. Phys. Lett. 2007, 90 (18), 181930.

67. Yoshida, H.; Sato, N. Grazing-Incidence X-Ray Diffraction Study of Pentacene Thin Films with the Bulk Phase Structure. Appl. Phys. Lett. 2006, 89 (10), 101919.

68. Siegrist, T.; Besnard, C.; Haas, S.; Schiltz, M.; Pattison, P.; Chernyshov, D.; Batlogg, B.; Kloc, C. A Polymorph Lost and Found: The High-Temperature Crystal Structure of Pentacene. $A d v$. Mater. 2007, 19 (16), 2079-2082. 
69. Campbell, R. B.; Robertson, J. M.; Trotter, J. The Crystal Structure of Hexacene, and a Revision of the Crystallographic Data for Tetracene. Acta Cryst. 1962, 15 (3), 289-290.

70. Nabok, D.; Puschnig, P.; Ambrosch-Draxl, C.; Werzer, O.; Resel, R.; Smilgies, D.-M. Crystal and Electronic Structures of Pentacene Thin Films from Grazing-Incidence X-Ray Diffraction and First-Principles Calculations. Phys. Rev. B 2007, 76 (23), 235322.

71. Schiefer, S.; Huth, M.; Dobrinevski, A.; Nickel, B. Determination of the Crystal Structure of Substrate-Induced Pentacene Polymorphs in Fiber Structured Thin Films. J. Am. Chem. Soc. 2007, $129(34), 10316-10317$.

72. Pachmajer, S.; Jones, A. O. F.; Truger, M.; Röthel, C.; Salzmann, I.; Werzer, O.; Resel, R. Self-Limited Growth in Pentacene Thin Films. ACS Appl. Mater. Interfaces 2017, 9 (13), $11977-11984$.

73. Moser, A.; Flesch, H.-G.; Neuhold, A.; Marchl, M.; Ausserlechner, S. J.; Edler, M.; Griesser, T.; Haase, A.; Smilgies, D.-M.; Jakabovič, J.; et al. Crystallization of Pentacene Thin Films on Polymeric Dielectrics. Synth. Met. 2012, 161 (23-24), 2598-2602.

74. Yang, H.; Ling, M.-M.; Yang, L. Temperature-Dependent Pentacene Nanostructures on Hydrophobic Gate-Dielectrics Correlated with Charge Carrier Mobilities. J. Phys. Chem. C 2007, $111(34), 12508-12511$.

75. Yu, X.; Yu, J.; Huang, W.; Zhang, L.; Zeng, H. Source/Drain Electrodes Contact Effect on the Stability of Bottom-Contact Pentacene Field-Effect Transistors. AIP Adv. 2012, 2 (2), 022113. 
76. Knipp, D.; Benor, A.; Wagner, V.; Muck, T. Influence of Impurities and Structural Properties on the Device Stability of Pentacene Thin Film Transistors. J. Appl. Phys. 2007, 101 (4), 044504.

77. Cipolloni, S.; Mariucci, L.; Valletta, A.; Simeone, D.; Angelis, F. D.; Fortunato, G. Aging Effects and Electrical Stability in Pentacene Thin Film Transistors. Thin Solid Films 2007, 515 (19), 7546-7550.

78. Angelis, F. D.; Cipolloni, S.; Mariucci, L.; Fortunato, G. Aging Effects in Pentacene ThinFilm Transistors: Analysis of the Density of States Modification. Appl. Phys. Lett. 2006, 88 (19), 193508.

79. Li, D.; Borkent, E.-J.; Nortrup, R.; Moon, H.; Katz, H.; Bao, Z. Humidity Effect on Electrical Performance of Organic Thin-Film Transistors. Appl. Phys. Lett. 2005, 86 (4), 042105.

80. Jurchescu, O. D.; Baas, J.; Palstra, T. T. M. Electronic Transport Properties of Pentacene Single Crystals upon Exposure to Air. Appl. Phys. Lett. 2005, 87 (5), 052102.

81. Qiu, Y.; Hu, Y.; Dong, G.; Wang, L.; Xie, J.; Ma, Y. H2O Effect on the Stability of Organic Thin-Film Field-Effect Transistors. Appl. Phys. Lett. 2003, 83 (8), 1644-1646.

82. Angelis, F. D.; Gaspari, M.; Procopio, A.; Cuda, G.; Fabrizio, E. D. Direct Mass Spectrometry Investigation on Pentacene Thin Film Oxidation upon Exposure to Air. Chem. Phys. Lett. 2009, 468 (4-6), 193-196. 
83. Maliakal, A.; Raghavachari, K.; Katz, H.; Chandross, E.; Siegrist, T. Photochemical Stability of Pentacene and a Substituted Pentacene in Solution and in Thin Films. Chem. Mater. 2004, $16(24), 4980-4986$.

84. Angelis, F. D.; Das, G.; Fabrizio, E. D. Analysis of the Interactions between Pentacene Film and Air Molecules by Means of Raman Spectroscopy. Chem. Phys. Lett. 2008, 462 (4-6), $234-237$.

85. Koch, N.; Salzmann, I.; Johnson, R. L.; Pflaum, J.; Friedlein, R.; Rabe, J. P. Molecular Orientation Dependent Energy Levels at Interfaces with Pentacene and Pentacenequinone. Org. Electron. 2006, 7 (6), 537-545.

86. Jurchescu, O. D.; Baas, J.; Palstra, T. T. M. Effect of Impurities on the Mobility of Single Crystal Pentacene. Appl. Phys. Lett. 2004, 84 (16), 3061-3063.

87. Salzmann, I.; Nabok, D.; Oehzelt, M.; Duhm, S.; Moser, A.; Heimel, G.; Puschnig, P.; Ambrosch-Draxl, C.; Rabe, J. P.; Koch, N. Structure Solution of the 6,13-Pentacenequinone Surface-Induced Polymorph by Combining X-Ray Diffraction Reciprocal-Space Mapping and Theoretical Structure Modeling. Cryst. Growth Des. 2011, 11 (2), 600-606.

88. Salzmann, I.; Duhm, S.; Opitz, R.; Rabe, J. P.; Koch, N. Impact of Low 6,13Pentacenequinone Concentration on Pentacene Thin Film Growth. Appl. Phys. Lett. 2007, 91 (5), 051919.

89. Shioya, N.; Shimoaka, T.; Hasegawa, T. Fringe and Noise Reductions of pMAIRS Spectra Using Principal Component Analysis. Anal. Sci. 2017, 33 (1), 117-120. 
90. Norimoto, S.; Morimine, S.; Shimoaka, T.; Hasegawa, T. Analysis of the Surface Coverage of a Self-Assembled Monolayer of Octadecyl Silane on a $\mathrm{Si}(100)$ Surface by Infrared External-Reflection Spectroscopy. Anal. Sci. 2013, 29 (10), 979-984.

91. Parisse, P.; Bussolotti, F.; Passacantando, M.; Ottaviano, L. 3D Island Growth of 6,13 Pentacenequinone on Silicon Oxide and Gold. J. Non. Cryst. Solids 2010, 356 (37-40), 20792082.

92. Hwang, D. K.; Kim, K.; Kim, J. H.; Im, S.; Jung, D.-Y.; Kim, E. Structural and Optical Properties of 6,13-Pentacenequinone Thin Films. Appl. Phys. Lett. 2004, 85 (23), 5568-5570.

93. Kamura, Y.; Shirotani, I.; Inokuchi, H.; Maruyama, Y. Absorption Spectra of Oriented and Amorphous Naphthacene and Pentacene Films. Chem. Lett. 1974, 3 (6), 627-630. 